\title{
A regional analysis of payer and provider views on cholesterol management: PCSK9 inhibitors as an illustrative alignment model
}

\author{
Dana McCormick, RPh; Deepak L Bhatt, MD, MPH, FACC, FAHA, FSCAI, FESC; Harold E Bays, MD, \\ FOMA, FTOS, FACC, FACE, FASPC, FNLA; Pam R Taub, MD, FACC; Kim A Caldwell, RPh; \\ Chris K Guerin, MD, FACE; Jeff Steinhoff, MD, FACC; Zahid Ahmad, MD; Rubina Singh, PharmD, BCPS; \\ Kathleen Moreo, RN-BC, BSN, BHSA, CCM, CDMS; Jeffrey Carter, PhD; Cherilyn L Heggen, PhD; and Tamar Sapir, PhD
}

\section{What is already known about this subject}

- Patients with inadequately controlled, high, or very high levels of lowdensity lipoprotein cholesterol (LDL-C) face multiple barriers in accessing optimal treatment.

- In eligible patients, challenges to the use of proprotein convertase subtilisin/kexin type 9 enzyme inhibitors (PCSK9i) include insufficient authorization documentation and high-payer rejection rates.

- Previous efforts to address drug access and prior authorization (PA) barriers through development and consensus approval of a standardized PA form have had limited uptake.

\section{What this study adds}

- This study identified access barriers that persisted despite reductions in pricing and publishing of clinical data on cardiovascular outcomes.

- Solutions identified during this study included recommendations for development and dissemination of a standardized PA form, educational programs for payers and providers on PA documentation processes and updated treatment guidelines, and patient educational programs on the importance of treatment adherence.

\section{Author affiliations}

Dana McCormick, RPh, Blue Cross Blue Shield of Texas, Richardson, TX; Deepak L Bhatt, MD, MPH, FACC, FAHA, FSCAI, FESC, Brigham and Women's Hospital Heart and Vascular Center and Harvard Medical School, Boston, MA; Harold E Bays, MD, FOMA, FTOS, FACC, FACE, FASPC, FNLA, Louisville Metabolic and Atherosclerosis Research Center, Louisville, KY; Pam R Taub, MD, FACC, Division of Cardiovascular Medicine, University of California San Diego School of Medicine; Kim A Caldwell, RPh, Texas Star Healthcare Consulting, McKinney; and Chris K Guerin, MD, FACE, Tri-City Medical Center and University of California San Diego School of Medicine. Jeff Steinhoff, MD, FACC, Largo Medical Center, Largo, FL; HCA Healthcare/USF Morsani College of Medicine, Tampa, FL; and Nova Southeastern University, Davie, FL. Zahid Ahmad, MD, Division of Nutrition and Metabolic Disease, UT Southwestern Medical Center, Dallas, TX, and Rubina Singh, PharmD, BCPS, AMCP, Alexandria, VA. Kathleen Moreo, RN-BC, BSN, BHSA, CCM, CDMS; Jeffrey Carter, PhD; Cherilyn L Heggen, PhD; and Tamar Sapir, PhD, PRIME Education, Fort Lauderdale, FL.

\section{AUTHOR CORRESPONDENCE:}

Cherilyn L Heggen, 954.718.6055,

c.heggen@primeinc.org 


\section{ABSTRACT}

BACKGROUND: Multiple barriers exist for appropriate use of the proprotein convertase subtilisin/kexin type 9 enzyme inhibitors (PCSK9i) in patients with atherosclerotic cardiovascular disease (ASCVD) or familial hypercholesterolemia (FH) with inadequately controlled hypercholesterolemia despite standard therapies. Among these barriers, high payer rejection rates and inadequate prior authorization (PA) documentation by providers hinder optimal use of PCSK9i.

OBJECTIVES: To (a) identify and discuss provider and payer discordances on barriers to authorization and use of PCSK9i based on clinical and real-world evidence and (b) align understanding and application of clinical, cost, safety, and efficacy data of PCSK9i.

METHODS: Local groups of 3 payers and 3 providers met in 6 separate locations across the United States through a collaborative project of AMCP and PRIME Education. Responses to selected pre- and postmeeting survey questions measured changes in attitudes and beliefs regarding treatment barriers, lipid thresholds for considering PCSK9i therapy, and tactics for improving PA processes. Statistical analysis of inter- and intragroup changes in attitudes were performed by Cox proportional hazards test and Fisher's exact test for $<5$ variables.

RESULTS: The majority of providers and payers (67\%-78\%) agreed that high patient copayments and inadequate PA documentation were significant barriers to PCSK9i usage. However, payers and providers differed on beliefs that current evidence does not support PCSK9i cost-effectiveness ( $6 \%$ providers, $56 \%$ payers; $P=0.003$ ) and that PA presents excessive administrative burden ( $72 \%$ providers, $44 \%$ payers; $P=0.09$ ) Average increases pre- to postmeeting were noted in provider beliefs that properly documented PA forms expedite access to PCSK9i (22\%-50\% increase) and current authorization criteria accurately distinguish patients who benefit most from PCSK9i (6\%-22\%). Payers decreased in their belief that current authorization criteria accurately distinguish benefiting patients (72\%-50\%). Providers and payers increased in their belief that PCSK9i are cost-effective (44\%-61\% and $28 \%-50 \%$, respectively) and were more willing to consider PCSK9i at the low-density lipoprotein cholesterol threshold of $>70 \mathrm{mg} / \mathrm{dL}$ for patients with ASCVD (78\%-83\% and $44 \%-67 \%$, respectively) or $\mathrm{FH}$ (22\%-39\% and $22 \%-33 \%$ ). Payers were more agreeable to less stringent PA requirements for patients with $\mathrm{FH}(33 \%-72 \%, \mathrm{P}=0.019)$ and need for standardized $\mathrm{PA}$ requirements $(50 \%-83 \%, P=0.034)$; these considerations remained high (89\%) among providers after the meeting. Most participants supported educational programs for patient treatment adherence (83\%) and physician/staff PA processes (83\%-94\%).

CONCLUSIONS: Provider and payer representatives in 6 distinct geographic locations provided recommendations to improve quality of care in patients eligible for PCSK9i. Participants also provided tactical recommendations for streamlining PA documentation processes and improving awareness of PCSK9i cost-effectiveness and clinical efficacy. The majority of participants supported development of universal, standardized patient eligibility criteria and PA forms.
Multiple atherosclerosis cardiovascular disease (ASCVD) outcomes studies support the importance of early and sustained lowering of low-density lipoprotein cholesterol (LDL-C) levels to reduce lifetime ASCVD risk. ${ }^{1,2}$ Current lipid treatment guidelines recommend high-intensity statin treatment in patients at high ASCVD risk, with or without other lipid-modifying therapies, such as ezetimibe or the monoclonal antibody proprotein convertase subtilisin/ kexin type 9 enzyme inhibitors (PCSK9i). However, among these high-risk ASCVD patients, less than $25 \%$ of patients achieve LDL-C threshold treatment levels of $<70 \mathrm{mg} / \mathrm{dL}^{3}$

Similarly, patients with heterozygous or homozygous forms of familial hypercholesterolemia $(\mathrm{FH})$ with high untreated baseline LDL-C levels (typically $>190 \mathrm{mg} / \mathrm{dL}$ in heterozygous $\mathrm{FH}$ or $>400 \mathrm{mg} / \mathrm{dL}$ in homozygous $\mathrm{FH}$ ) also have a high lifetime risk for ASCVD events. Many do not achieve LDL-C $<70 \mathrm{mg} / \mathrm{dL}$, or even $<100 \mathrm{mg} / \mathrm{dL}$, despite high-intensity statins and maximally tolerated statins, since statin and ezetimibe treatment may not be sufficient to lower LDL-C levels in patients with very high baseline cholesterol levels. ${ }^{4-6}$

Based on results of earlier clinical trials, outcomes trials, meta-analysis, and real-world evidence, the 2 approved PCSK9i, alirocumab and evolocumab, have demonstrated significant reductions $(\geq 50 \%-60 \%)$ in LDL-C levels and incident CV events (e.g., myocardial infarction, stroke, and coronary revascularization or unstable angina) in high-risk patients with elevated LDL-C who fail to meet their lipid treatment goals with standard of care regimens and those who are statin intolerant. ${ }^{7-16}$ These include patients with ASCVD and significant risk factors such as type 2 diabetes or $\mathrm{FH}$. If LDL-C goals are not achieved with maximally tolerated statins, the 2018 AHA/ACC Guideline on the Management of Blood Cholesterol recommends a PCSK9i as the preferred add-on, nonstatin agent for patients with clinical ASCVD and comorbidities requiring $>25 \%$ additional lowering of LDL-C. ${ }^{17,18}$

Despite these guideline recommendations, patients meeting eligibility criteria for PCSK9i have high payer rejection rates, contributing to the underuse of PCSK9i in patients at high ASCVD risk or FH who have not achieved LDL-C goals. ${ }^{19-21}$ PCSK9i prescription rates of less than $<0.5 \%$ are reported for patients with dyslipidemia, coronary artery disease, or coronary heart disease and untreated LDL $\geq 130 \mathrm{mg} / \mathrm{dL}{ }^{19,21}$ Hess et al. (2017) reported PCSK9i prior authorization (PA) rejection rates ranging from 15\%-22\% in patients with LDL-C levels of 70-250 mg/dL and overall rejection rate of $53 \%$ in patients with or without ASCVD diagnosis. ${ }^{19}$ PCSK9i therapy may also be underused in patients with a history of acute ischemic stroke or transient ischemic attack and peripheral arterial disease. ${ }^{22}$ Extended 
periods for approval or payer rejection from time of initial diagnosis also hinder effective and timely use of these therapies. ${ }^{23}$

Multiple challenges exist in gaining access to PCSK9i therapy for many patients with inadequately controlled lipid levels or at high risk for ASCVD. These barriers include PA rejections due to (a) lack of required full documentation from providers supporting approved indicated use, (b) laboratory value criteria, (c) step and concomitant lipid-altering therapy requirements, and (d) criteria for continuation of therapy. ${ }^{24,25}$ Step and concomitant lipid-altering therapy requirements may include multiple trials of statins or simultaneous statin administration for PCSK9i therapy approval. Additional barriers are listed in Supplementary Table 1 (available in online article), which highlight the effect of provider perceptions of administrative burden, inadequate PA documentation, lack of consistent guidelines for lipid thresholds, and provider/payer knowledge of published outcomes research on treatment decisions and authorization.

To address these treatment gaps, 2 town hall meetings were sponsored by the American Society for Preventive Cardiology (ASPC) in 2017, which included participation by expert providers from the American College of Cardiology, National Lipid Association, American Association of Clinical Endocrinologists (AACE), and FH Foundation to identify and implement viable solutions aimed to improve use of PCSK9i therapies. ${ }^{25}$ These sessions facilitated the development of a standardized PCSK9i authorization template from the ASPC, which was endorsed by the AACE. However, there has been limited uptake of this template, prompting the need for additional initiatives to address persistent barriers contributing to undertreatment.

In 2019, AMCP hosted a partnership forum for payers to discuss optimization of PA for appropriate medication selection. ${ }^{26}$ The goals of the forum were to identify possible improvements in authorization efficiencies, address administrative burdens, provide technological administrative solutions, increase understanding of the clinical and economic value of PA and step therapy, collect and disseminate clinical and real-world data supporting the clinical and economic value of PA, and collect and disseminate best practices for authorization denials and appeals.

Previous forums provided separate input from providers and payers on barriers and proposed solutions for improving authorization processes for medication selection. However, limited face-to-face forums had been convened for providers and payers to discuss beliefs regarding administrative difficulties with PA, suitability of current authorization criteria for identifying appropriate patients, and gaps in physician and payer education on authorization and treatment guidelines for hypercholesterolemia. Since providers and payers are working toward the same goals of achieving optimal treatment outcomes and quality of life for patients, it was important to identify key issues affecting appropriate and timely use of PCSK9i for patients at greatest need and to proactively develop collaborative solutions in a live forum with consideration for provider and payer perspectives on the issues.

This report details the outcomes of 6 face-to-face focus group meetings in 2019 that hosted expert physician providers and key pharmacy representatives from payer organizations. The topics included discussions of barriers and solutions to improving undermanagement of hypercholesterolemia, alignment of practice with evidencebased guidelines, and identification of differences between groups in different geographic locations and changes in attitude and beliefs regarding key topics during the course of the meetings. Key outcomes of these discussions were presented to payer representatives at the 2019 AMCP Nexus meeting for payers to examine and compare discordances and concordances between providers and payers in 6 distinct U.S. locations and to discuss the feasibility of a universal PA form.

\section{Methods}

The initiative aimed to assess discordances in large cities (population $>500,000$ individuals based on U.S. Census data) within U.S. states with rates of preventable cardiovascular events exceeding 950 per 100,000 adults aged 18 years and older. ${ }^{27,28}$ Based on these criteria, a series of 6 live focus groups were conducted between August and September 2019 in San Diego, CA; Dallas, TX; Atlanta, GA; Tampa/Saint Petersburg, FL; Louisville, KY; and New York, NY. Each face-to-face meeting was composed of 3 cardiologists or lipidologists who represented large clinical practices providing care for $>100$ patients with hypercholesterolemia per month in the city where the meeting was held and 3 payer leaders from major health care management organizations, which provided coverage to $>1,500$ patients per month with hypercholesterolemia in the same geographic area. The overarching goals of the meetings were to uncover root causes for practice discordances with evidence-based guidelines and data and undertreatment of hypercholesterolemia, as well as to explore opportunities to improve interpretation of the evidence and understanding of the clinical, cost, safety, and efficacy of PCSK9i.

Participants completed premeeting paper surveys consisting of 33 items (providers) or 34 items (payers) that were designed to be completed in approximately 15 minutes. Based on a review of recently published literature, we 
identified potential barriers that informed the development of the survey items (Supplementary Table 1, available in online article). The first 2 items asked participants to indicate, from closed-ended lists, the type of organization in which they work (providers and payers) and their professional role (payers). The next question asked providers and payers to report the number of patients/organization members with hypercholesterolemia they manage on a weekly basis. The next 4 survey items asked respondents to estimate what percentage of their patients/organization members have barriers that limit them from achieving LDL-C goals, such as statin intolerance or side effects, nonadherence, $\mathrm{FH}$, and insurance denial. Participants were asked to report the most common reason for denial of an initial PCSK9i therapy request and to estimate the number of hours per week physician practice staff devote to PAs involving PCSK9i therapies.

A Likert scale from 1 (strongly disagree) to 5 (strongly agree) was used to assess level of agreement with statements about the effect of PCSK9i therapies on reducing the risk of major cardiovascular events by clinically significant magnitudes; cost-effectiveness; ability of current PA processes to accurately distinguish appropriate patients; appropriateness of the amount and type of information requested in current PA processes; current rates of PCSK9i therapy denials; whether eligible patients will have prompt access to treatment if PA paperwork is completed according to health plan requirements; and if patients who experience adverse events on an initial statin should be rechallenged with additional statins (write in number) before being eligible for PCSK9i therapy.

Through closed-ended lists, participants were asked to indicate LDL-C thresholds that should trigger PCSK9i therapy approval for eligible patients with ASCVD or FH. Closed-ended lists were also used to assess how respondents would order management actions for patients who do not achieve LDL-C goals. Using a Likert scale from 1 (not a barrier at all) to 5 (extremely significant barrier), participants were asked to rate potential barriers to therapy access.

Perceptions of the potential effectiveness of strategies to overcome barriers were assessed on a Likert scale from 1 (not effective at all) to 5 (extremely effective). The survey concluded with an open-text item that asked payers and providers to list 3 additional strategies that may offer effective solutions for overcoming barriers to appropriate and prompt access to PCSK9i therapies.

Following the program, participants completed paper postmeeting surveys that consisted of 17 questions designed to be completed in 10 minutes. This postmeeting survey repeated questions from the presurvey to measure changes (via Likert scales from 1 to 5) in agreement and perceptions on potential effectiveness of strategies to overcome barriers that payers and providers may be positioned to address through collaborative initiatives.

Statistical analysis between group responses to premeeting and postmeeting survey questions were calculated by Cox proportional or Fisher's exact tests. Audio recordings from 6 live focus groups were transcribed and imported into Dedoose, a web application for mixed methods analysis. Thematic analysis was conducted using grounded theory to identify, analyze, and describe all themes within the dataset. In the first cycle of coding, structural codes based on questions from the focus group moderator guide were applied to index the transcripts. Descriptive and in vivo codes were applied inductively to summarize key ideas discussed by participants. The initial codes were refined to create a structured code list that was applied to all of the transcripts during subsequent stages of coding and analysis to generate categories and themes. Code frequencies and themes were also analyzed to identify key areas of alignment and discordance.

\section{Results}

Eighteen providers and 18 payers ( 3 each per meeting) attended the focus group meetings. The 18 providers consisted of cardiologists and/or lipidologists who provided care for more than 25 patients with hypercholesterolemia per week. Payers who participated in the focus group meetings reported roles in managed care organizations or health plans (12 payers) or pharmacy benefit management organizations (6 payers) and managing more than 400 patients per week (Table 1).

\section{PRESURVEY BARRIERS TO USE OF PCSK9i THERAPIES}

Figure 1 shows the percentage of premeeting responses by providers and payers to the 5 barriers to uptake and use of PCSK9i assessed in the survey. High percentages of participants reported that high copayments (72\% payers and $67 \%$ providers) and insufficient PA documentation (78\% payers and $72 \%$ providers) were barriers. Compared with payers, there was a trend towards more providers reporting that PCSK9i PA documentation was excessive and burdensome to physicians and office staff, although the difference did not reach statistical significance. Compared with providers, payers were significantly $(P=0.003)$ more likely to report that insufficient evidence existed for PCSK9i cost-effectiveness; however, low percentages of providers $(11 \%)$ and payers (17\%) reported insufficient evidence that PCSK9i therapies reduced risks of major cardiovascular events as a barrier. 


\begin{tabular}{|c|c|}
\hline & Participants, \% (n) \\
\hline \multicolumn{2}{|l|}{ Work organizations } \\
\hline Managed care organization or health plan & $33.3 \quad(12)$ \\
\hline Pharmacy benefit management organization & $16.7 \quad(6)$ \\
\hline Academic medical center & $22.2 \quad(8)$ \\
\hline Private practice & $13.9 \quad(5)$ \\
\hline Hospital & $11.1 \quad(4)$ \\
\hline Community-based clinic & $2.8 \quad(1)$ \\
\hline \multicolumn{2}{|l|}{ Professional roles } \\
\hline Pharmacy director & $13.9 \quad(5)$ \\
\hline Clinical pharmacist & $13.9 \quad(5)$ \\
\hline Drug utilization/formulary management & $11.1 \quad(4)$ \\
\hline Account manager & $2.8 \quad(1)$ \\
\hline Health economics and outcomes research & $2.8 \quad(1)$ \\
\hline Pharmacy and therapeutics committee member & $5.6 \quad(2)$ \\
\hline Cardiologist & $30.6 \quad(11)$ \\
\hline Lipidologist & $19.4 \quad(7)$ \\
\hline
\end{tabular}

\section{QUALITATIVE REVIEW OF FOCUS GROUP TRANSCRIPTS}

Through qualitative review of discussion transcripts, across all focus groups, providers expressed frustration with the complexity of completing PA forms. Even with physician and staffing time investment, providers had an expectation of high rejection rates. In the discussions, some payers indicated that the information provided on the PA forms is often insufficient for approval, while many providers stated that approvals required too much information. Time restrictions for providers and staff to complete the extensive PA requirements were considered significant hindrances to approval. Additional topics discussed during individual meetings collected through qualitative review of the transcripts are listed in Supplementary Table 2 (available in online article).

In the discussions, payers and providers concurred on the benefits of an agreed upon, standardized PA form to simplify the approval process and which would be uniform among all payers. Because of the uniqueness of the population, 1 participant suggested a separate PA form for $\mathrm{FH}$ patients. Providers generally reported that there is a need to treat hypercholesterolemia more aggressively and that strong clinical data on safety and risk reduction and revised treatment guidelines support the use of PCSK9i when more robust LDL-C reduction is necessary. Payers proposed revising $\mathrm{PA}$ form requirements and assigning dedicated care coordinators to provider offices. These care coordinators would offer education to providers on long-term management with PCSK9. One participant recommended close collaboration with specialty pharmacy managers.

\section{ASSESSING ATTITUDES AND BELIEFS OF PAYERS AND PROVIDERS BEFORE AND AFTER FOCUS GROUP DISCUSSIONS}

Analysis of participant pre- and postsurvey responses did not reveal any statistically significant changes in the attitude/belief statements (Table 2). However, numerical increases were noted in provider and payer agreement with the following statements:

- If PA paperwork is completed according to health plan requirements, eligible patients will have prompt access to PCSK9i therapies $(22 \%-50 \%$, providers; $83 \%-94 \%$, payers)

- PCSK9i therapies are cost-effective $(44 \%-61 \%$, providers; $28 \%-50 \%$, payers)

- For eligible patients, current rates of PCSK9i therapy denials are excessively high (6\%-17\%, payers)

- The amount and type of information requested in current PA processes is appropriate for effective use of PCSK9i therapies (22\%-17\%, providers; 67\%-50\%, payers)

- Current PA processes accurately distinguish patients who would benefit most from PCSK9i therapies (6\%-22\%, providers; $72 \%-50 \%$, payers)

- For eligible patients, PCSK9i therapies reduce risks of major cardiovascular events by clinically significant magnitudes (83\%-94\%, payers).

Despite some numerical gains, comparisons of survey responses between providers and payers indicated that agreement between providers and payers remained low on approval success with appropriately completed PA paperwork (50\% providers vs. 94\% payers), high rejection rates ( $56 \%$ vs. $17 \%)$, PA process requirements (17\% vs. $50 \%)$, and ability of PA to accurately identify patients who could most benefit from PCSK9i (22\% vs. 50\%), although these differences were not statistically significant.

Conversely, after the meetings, responses were numerically closer in agreement on "PCSK9i therapies are cost-effective" (61\% providers vs. $50 \%$ payers) and "for eligible patients, PCSK9i therapies reduce risks of major cardiovascular events by clinically significant magnitudes" (94\% vs. 94\%).

\section{LIPID THRESHOLDS}

During the meetings, numerical shifts were observed among providers and payers related to minimum thresholds for considering PCSK9i therapy, although these changes were not statistically significant. For eligible patients with 


\section{FIGURE 1 Premeeting Responses to Perceptions of Key Barriers to PCSK9i Use ${ }^{a}$}

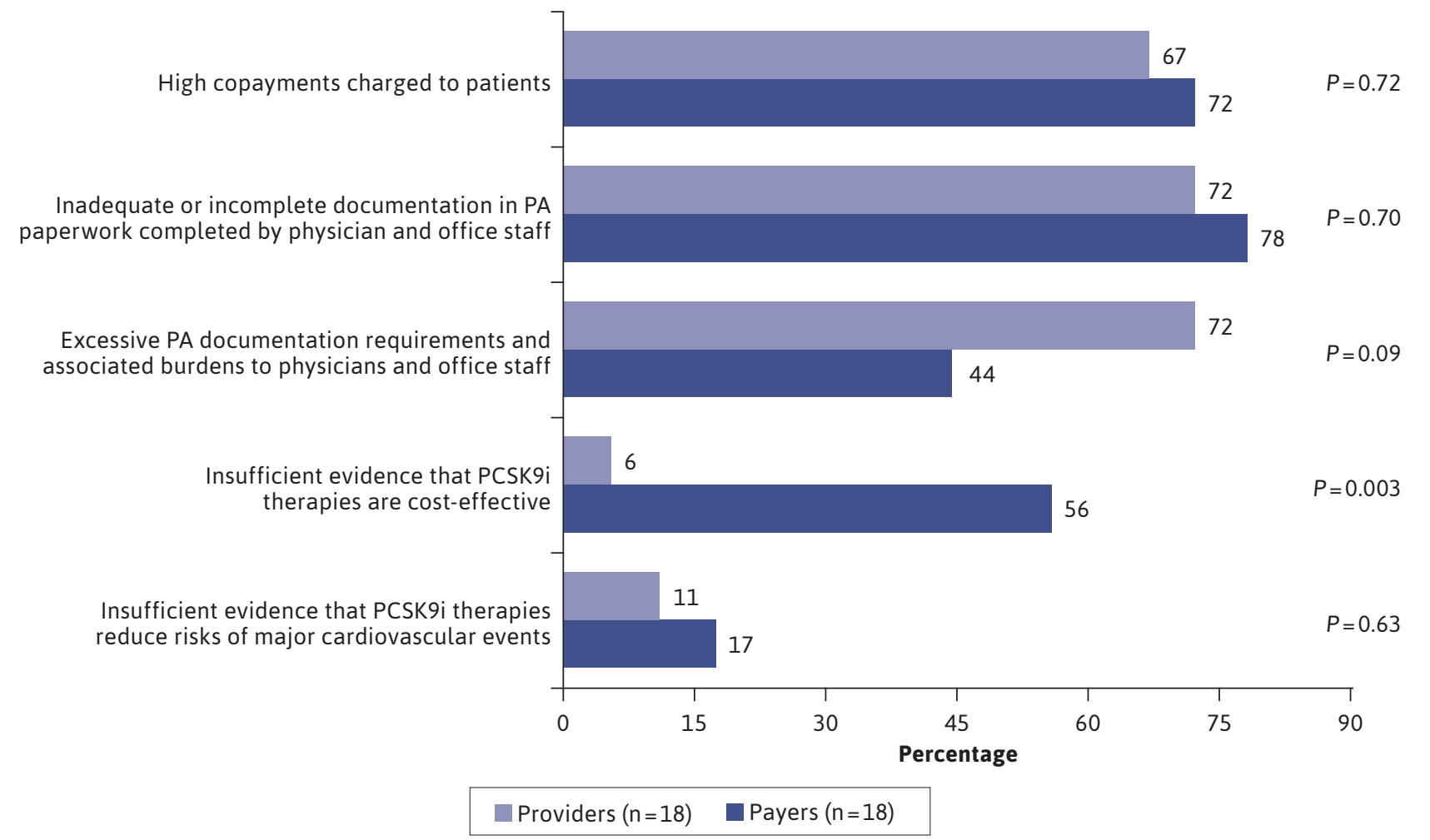

aPooled ratings of 4 (agree) and 5 (strongly agree) on a Likert scale.

$P A=$ prior authorization; $P C S K 9 i=$ proprotein convertase subtilisin/kexin type 9 enzyme inhibitor.

ASCVD, higher percentages of providers (78\% premeeting to $83 \%$ postmeeting) and payers $(44 \%-67 \%)$ reported that they would consider $70 \mathrm{mg} / \mathrm{dL}$ as a minimum threshold for eligible patients with ASCVD (Figure 2). Similarly, higher percentages of providers (22\% premeeting to $39 \%$ postmeeting) and payers $(22 \%-33 \%)$ reported that they would consider PCSK9i therapy for FH patients with LDL-C $>70 \mathrm{mg} / \mathrm{dL}$. Postmeeting responses indicated that none of the participants favored an initiation threshold LDL-C level of $>130$ $\mathrm{mg} / \mathrm{dL}$ for ASCVD patients and $>160 \mathrm{mg} / \mathrm{dL}$ for $\mathrm{FH}$ patients.

\section{TACTICAL STRATEGIES FOR IMPROVING ACCESS TO PCSK9i THERAPIES}

Table 3 lists the pre- and postmeeting responses to 8 tactical considerations presented for improving PA processes and educational programming for patients and providers. Following the meetings, statistically significant increases were noted in the percentages of payers who supported separate, less arduous PA requirements for $\mathrm{FH}$ patients
(33\% premeeting to $72 \%$ postmeeting; $\mathrm{P}=0.019$ ) and standardized PA requirements and forms $(50 \%-83 \% ; P=0.034)$.

Numerical increases were observed in the percentage of payers who prioritized creating payer websites to streamline PA and appeals documentation (61\% premeeting to 67\% postmeeting), educational programs to support patients in partnering with providers to address PA requirements and denials (61\%-72\%), and to support physicians and office staff in preparing necessary PA documentation (78\%-94\%), although these increases were not statistically significant.

Percentages of payers decreased numerically for assigning dedicated case managers in health plans to communicate directly with physician offices about PA processes ( $72 \%$ premeeting to $50 \%$ postmeeting), health plan coverage for $\mathrm{FH}$ genetic testing when required for PA (56\%-44\%), and educational programs to support patients in improving treatment adherence (100\%-83\%).

For providers and payers, percentages increased for creating payer websites to streamline PA and appeals 


\section{TABLE 2 Attitudes and Beliefs Relating to Approval for and Effectiveness of PCSK9i Therapies}

\begin{tabular}{|c|c|c|c|c|c|c|c|c|}
\hline \multirow[b]{2}{*}{ Survey Question } & \multicolumn{2}{|c|}{ Providers $(n=18)$} & \multirow{2}{*}{$\begin{array}{l}\text { Absolute } \\
\% \text { Change }\end{array}$} & \multirow{2}{*}{$\begin{array}{c}P \\
\text { Value }\end{array}$} & \multicolumn{2}{|c|}{ Payers $(n=18)$} & \multirow{2}{*}{$\begin{array}{l}\text { Absolute } \\
\% \text { Change }\end{array}$} & \multirow{2}{*}{$\begin{array}{c}P \\
\text { Value }\end{array}$} \\
\hline & Presurvey & Postsurvey & & & Presurvey & Postsurvey & & \\
\hline $\begin{array}{l}\text { If PA paperwork is completed according to } \\
\text { health plan requirements, eligible patients will } \\
\text { have prompt access to PCSK9i therapies }\end{array}$ & $22.2^{\mathrm{a}}(4)$ & 50.0 & $27.8 \quad(5)$ & 0.16 & $83.3(15)$ & $94.4(17)$ & 11.1 & 0.60 \\
\hline $\begin{array}{l}\text { For eligible patients, current rates of PCSK9i } \\
\text { therapy denials are excessively high }\end{array}$ & $55.6(10)$ & $55.6(10)$ & $0.0 \quad(0)$ & 1.00 & $5.6(1)$ & $16.7 \quad(3)$ & $11.1(2)$ & 0.60 \\
\hline $\begin{array}{l}\text { The amount and type of information requested } \\
\text { in current PA processes is appropriate for } \\
\text { effective use of PCSK9i therapies }\end{array}$ & 22.2 (4) & $16.7 \quad(3)$ & $-5.5(-1)$ & 0.67 & $66.7(12)$ & 50.0 & $-16.7(-3)$ & 0.31 \\
\hline $\begin{array}{l}\text { Current PA processes accurately distinguish } \\
\text { patients who would benefit most from PCSK9i } \\
\text { therapies }\end{array}$ & $5.6(1)$ & 22.2 & 16.6 & 0.34 & $72.2(13)$ & 50.0 & $-22.2(-4)$ & 0.17 \\
\hline PCSK9i therapies are cost-effective & $44.4 \quad(8)$ & $61.1(11)$ & 16.7 (3) & 0.32 & $27.8 \quad(5)$ & $50.0 \quad(9)$ & 22.2. (4) & 0.17 \\
\hline $\begin{array}{l}\text { For eligible patients, PCSK9i therapies reduce } \\
\text { risks of major cardiovascular events by } \\
\text { clinically significant magnitudes }\end{array}$ & $94.4(17)$ & $94.4(17)$ & $0.0 \quad(0)$ & 1.00 & $83.3(15)$ & $94.4(17)$ & $11.1(2)$ & 0.60 \\
\hline \multicolumn{9}{|c|}{$\begin{array}{l}\text { Note: All results are \% (n). } \\
\text { apooled ratings of } 4 \text { (agree) and } 5 \text { (strongly agree) on a Likert scale. }\end{array}$} \\
\hline
\end{tabular}

documentation $(67 \%$ premeeting to $72 \%$ postmeeting, providers; $61 \%$ premeeting to $67 \%$ postmeeting, payers) and educational programs to support patients in partnering with providers on PA requirements $(61 \%-67 \%$, providers; $61 \%-72 \%$, payers), although these changes were not statistically significant.

From pre- to postmeeting, there was a numerical increase in the percentage of providers who supported creating payer websites to streamline PA and appeals documentation (67\% premeeting to $72 \%$ postmeeting), health plan coverage for $\mathrm{FH}$ genetic testing when required for PA (39\%-50\%), and educational programs to support patients in partnering with providers on PA requirements (61\%-67\%), while decreases were observed in the percentage of providers who prioritized separate, less arduous PA requirements for FH patients (100\%-89\%), standardized PA requirements and forms (94\%-89\%), and educational programs to support patients in improving treatment adherence (89\%-83\%). Percentages remained the same for assigning dedicated case managers in health plans to communicate directly with physician offices about PA processes and appeals to denials (83\% pre- and postmeeting) and educational programs to support physicians and office staff in preparing necessary PA documentation (83\% pre- and postmeeting).

\section{Discussion}

This analysis was derived from data collected from 6 meetings in distinct geographic locations across the United States that gathered expert providers in cardiovascular health care, teamed with pharmacy leaders representing major regional health care payer organizations, in face-toface focus group discussions. This analysis examined the attitudes and beliefs of providers and payers about barriers to appropriate use of PCSK9i therapies for patients with uncontrolled LDL-C and FH, ASCVD, or at high risk for ASCVD. The series of 6 meetings provided forums for robust discussion regarding metrics for measuring outcomes in identifying specific disparities and areas of consensus for solutions to address undertreatment of hypercholesterolemia.

Over the course of the meetings, which included faceto-face discussion among diverse group members with different perspectives, viewpoints of providers and payers tended to converge in substantive areas, with a greater appreciation and agreement regarding management of challenges. Meeting participants developed meaningful solutions to the identified barriers and discussed practical steps for potential implementation, which could then serve as a model for other PA constructs for future therapeutics.

Barriers to appropriate use of PCSK9i therapies identified in these meetings generally reflected the issues 


\section{FIGURE 2 Attitudes/Beliefs Relating to LDL-C Thresholds for Considering PCSK9i Therapy}

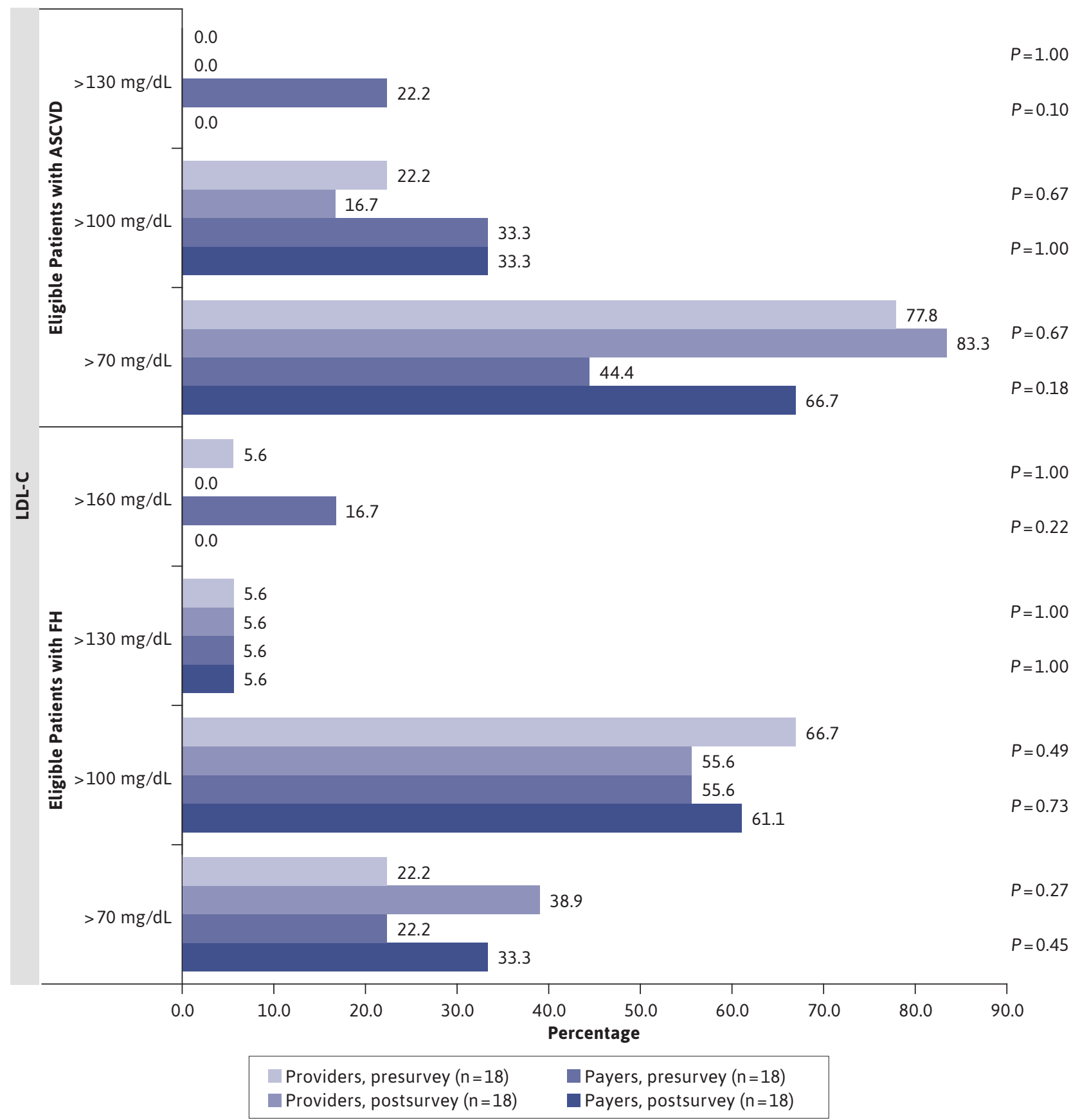

aPercentages of participants who selected LDL-C thresholds from closed-ended lists pre-and postsurvey.

$A S C V D=$ atherosclerotic cardiovascular disease; $F H=$ familial hypercholesterolemia; $L D L-C=l o w$-density lipoprotein cholesterol; $P C S K 9 i=$ proprotein convertase subtilisin/kexin type 9 enzyme inhibitor. 
Tactical Considerations for Improving Prior Authorization Processes and Educational Programming for Patients and Providers

\begin{tabular}{|c|c|c|c|c|c|c|c|c|}
\hline \multirow[b]{2}{*}{ Survey Question } & \multicolumn{2}{|c|}{ Providers $(n=18)$} & \multirow{2}{*}{$\begin{array}{l}\text { Absolute } \\
\% \text { Change }\end{array}$} & \multirow{2}{*}{$\begin{array}{c}P \\
\text { Value }\end{array}$} & \multicolumn{2}{|c|}{ Payers $(n=18)$} & \multirow{2}{*}{$\begin{array}{l}\text { Absolute } \\
\% \text { Change }\end{array}$} & \multirow{2}{*}{$\begin{array}{c}P \\
\text { Value }\end{array}$} \\
\hline & Presurvey & Postsurvey & & & Presurvey & Postsurvey & & \\
\hline $\begin{array}{l}\text { Assigning dedicated case managers in health } \\
\text { plans to communicate directly with physician } \\
\text { offices about PA processes and appeals to } \\
\text { denials }\end{array}$ & $83.3^{a}(15)$ & $83.3(15)$ & $0.0 \quad(0)$ & 1.00 & $72.2(13)$ & $50.0 \quad(9)$ & $-22.2(-4)$ & 0.17 \\
\hline $\begin{array}{l}\text { Creating payer websites to streamline PA and } \\
\text { appeals documentation for PCSK9i therapy }\end{array}$ & $66.7(12)$ & $72.2(13)$ & $5.5 \quad(1)$ & 0.72 & $61.1(11)$ & $66.7(12)$ & $5.6 \quad(1)$ & 0.73 \\
\hline $\begin{array}{l}\text { Health plan coverage for } \mathrm{FH} \text { genetic testing, } \\
\text { when required for PA }\end{array}$ & $38.9 \quad(7)$ & $50.0 \quad(9)$ & 11.1 & 0.50 & $55.6(10)$ & $44.4 \quad(8)$ & $-11.2(-2)$ & 0.51 \\
\hline $\begin{array}{l}\text { Separate, less arduous PA requirements for } \\
\text { patients with FH }\end{array}$ & 100.0 & $88.9(16)$ & $-11.1(-2)$ & 0.49 & $33.3 \quad(6)$ & $72.2(13)$ & $38.9 \quad(7)$ & 0.019 \\
\hline $\begin{array}{l}\text { Standardized PA requirements and forms } \\
\text { across health plan and payer organizations }\end{array}$ & 94.4 (17) & $88.9(16)$ & $-5.5(-1)$ & 0.55 & 50.0 & $83.3(15)$ & $33.3 \quad(6)$ & 0.034 \\
\hline $\begin{array}{l}\text { Educational programs to support patients in } \\
\text { improving adherence to their prescribed } \\
\text { lipid-lowering therapies }\end{array}$ & $88.9(16)$ & $83.3(15)$ & $-5.6(-1)$ & 0.63 & 100.0 & $83.3(15)$ & $-16.7(-3)$ & 0.23 \\
\hline $\begin{array}{l}\text { Educational programs to support patients } \\
\text { in partnering with providers to address PA } \\
\text { requirements and denials }\end{array}$ & $61.1(11)$ & $66.7(12)$ & $5.6 \quad(1)$ & 0.72 & $61.1(11)$ & $72.2(13)$ & $11.1 \quad(2)$ & 0.72 \\
\hline $\begin{array}{l}\text { Educational programs to support physicians } \\
\text { and office staff in preparing necessary } \\
\text { documentation for PA and appeals to denials }\end{array}$ & $83.3(15)$ & $83.3(15)$ & $0.0 \quad(0)$ & 1.00 & 77.8 (14) & $94.4(17)$ & $16.6 \quad(3)$ & 0.34 \\
\hline
\end{tabular}

Note: All results are $\%(n)$.

apooled ratings of 4 (effective) and 5 (extremely effective) on a Likert scale.

$\mathrm{FH}=$ familial hypercholesterolemia; $P A=$ prior authorization; $P C S K 9 i=$ proprotein convertase subtilisin/kexin type 9 enzyme inhibitor.

identified in town hall meetings of expert lipidologists that were organized by the ASPC in 2017.25 Key data from these meetings were reviewed during the national AMCP Nexus 2019 fall symposium, where payers focused discussion primarily on the clinical administrative burden placed on providers and their staff for completing the stringent and extensive amount of information required on PA forms, as well as on the frustration with and disproportionate amount of administrative time associated with denials and appeals processes.

Payers highlighted problems associated with inadequate PA process fulfillment by providers (inadequate documentation for necessary review, misunderstanding of process/criteria) responsible for PA denial rates and lack of follow through for appeals. Additional issues stemmed from (a) lack of standardization in PA requirements and documentation, (b) lack of resources among providers to tackle the administrative requirement, (c) perceived variability across clinical guidelines (payer and provider confusion), (d) high copay costs for some patients, and (e) lack of newer data on cost-effectiveness of PCSK9i. Following the
Nexus 2019 symposium, 46\% of the payers in attendance $(\mathrm{N}=82)$ identified "standardized PA requirements and forms across health plan and payer organizations" as 1 of their top 3 strategies for overcoming barriers to appropriate hypercholesterolemia care.

During the course of the meetings in 2019, there was a numerical shift in the attitudes of providers and payers regarding the threshold LDL-C levels at which PCSK9i therapy should be considered, with a postmeeting shift toward $>70 \mathrm{mg} / \mathrm{dL}$ in eligible patients with ASCVD and FH. This reflected a willingness to consider earlier intervention for patients not meeting their treatment goals, which, in practice, may assist in lifetime cardiovascular risk reduction. ${ }^{1,2}$ A systematic subgroup analyses of statin and PCSK9i trials demonstrated that PCSK9i therapies can provide reasonable monetary therapeutic value for secondary disease prevention when LDL-C is $\geq 70 \mathrm{mg} / \mathrm{dL}, \geq 100 \mathrm{mg} / \mathrm{dL}$ for primary prevention in patients with heterozygous $\mathrm{FH}$, or severe hypercholesterolemia with untreated LDL-C levels $\geq 220 \mathrm{mg} / \mathrm{dL}^{2}$ 
A recent analysis from ODYSSEY OUTCOMES found particular value in patients with $\mathrm{LDL}-\mathrm{C} \geq 100 \mathrm{mg} / \mathrm{dL}$ at current pricing. ${ }^{29-31}$ Guidelines from the American Association of Clinical Endocrinologists recommend an interventional target level of $<55 \mathrm{mg} / \mathrm{dL}$ LDL-C in patients at very high or extreme risk with progressive, established, or premature ASCVD and chronic kidney disease, type 2 diabetes, or $\mathrm{HeFH}^{32}$ Similar recommendations are included in the newest treatment guidelines from the European Society of Cardiology. ${ }^{33}$

Participants at the focus group meetings and AMCP symposium prioritized 2 goals for future actions aimed at meaningful solutions to appropriate use of PCSK9i therapies. The primary goal that was recommended was to collaboratively develop standardized PA criteria, a universal PA form, and universal PA process across all payers and U.S. regions as consistent with existing lipid treatment guidelines with input from providers and payers.

The secondary goal was to implement educational initiatives to teach providers and their staff how best to integrate PA documentation in the office/clinic workflow. Possible tactics included education of providers about real-world evidence, updated treatment guidelines, and revised costs for PCSK9i therapies. Recent data showing greater and faster prescription and use rates of PCSK9i therapies in eligible patients treated at hospitals with integrated specialty pharmacy clinics support recommendations for increased collaboration with pharmacy managers. ${ }^{34}$ Other solutions may be raised by increasing regional dialogue between payers and providers and quantifying the cost savings of early intervention.

Other potential solutions prioritized by participants to achieve optimal LDL-C management and access to appropriate treatment were (a) improved education on the recent updates to treatment guidelines, (b) education on patient adherence, and (c) increased recognition by payers of the significant cardiovascular risks in FH patients that are not adequately addressed by step statin and statin/ lipid-modifying therapy. In addition, the reductions in cardiovascularassociated health care costs that can be realized by earlier and/or broader use of PCSK9i therapies in eligible high-risk patients should be communicated to audiences in the medical community and payer organizations. ${ }^{2}$

\section{LIMITATIONS}

This study was limited by the small number of providers and payers present at each meeting, which prevents wider extrapolation of attitudes and strategic/tactical recommendations to larger provider/payer audiences. The overall sample size was small and not randomized, which affected the ability of the study to detect statistically significant changes. The study was also limited by the specific language of each survey question and the relatively short period of time given to each attendee to answer questions before and after the meetings.

\section{Conclusions}

Findings from this study highlight the need to focus time and resources of providers and payers on helping direct the most appropriate care to patients with hypercholesterolemia. To facilitate this objective, this effort illustrated an approach wherein providers and payers collaborated via face-to-face discussions on developing consensus solutions to administrative issues on PA documentation and appeals that affect patient eligibility, developing and disseminating standardized PA forms, and clarifying perceptions of costeffectiveness and efficacy of PCSK9i therapies based on current evidence.
Together, such an approach resulted in practical and actionable recommendations that will hopefully assist providers and payers to increase the quality of care for patients in greatest need for effective control of lipids. If successful, perhaps such a collaborative approach might prove effective for future therapeutics having similar PA challenges.

\section{DISCLOSURES}

The study reported in this article was part of a continuing education program funded by an independent educational grant awarded by Sanofi US and Regeneron Pharmaceuticals to PRIME Education. The grantor had no role in the study design, execution, analysis, or reporting. AMCP received grant funding from PRIME to assist in the study, as well as in writing the manuscript. McCormick, Bhatt, Bays, Taub, Caldwell, Guerin, Steinhoff, and Ahmad received an honorarium from PRIME for serving as faculty for the continuing education program. McCormick, Bhatt, Bays, Taub, Caldwell, Guerin, Steinhoff, and Ahmad were involved as participants in the study.

Bhatt discloses the following relationships: Advisory board: Cardax, CellProthera, Cereno Scientific, Elsevier Practice Update Cardiology, Level Ex, Medscape Cardiology, PhaseBio, PLx Pharma, Regado Biosciences; Board of directors: Boston VA Research Institute, Society of Cardiovascular Patient Care, TobeSoft; Chair: American Heart Association Quality Oversight Committee; Data monitoring committees: Baim Institute for Clinical Research (formerly Harvard Clinical Research Institute, for the PORTICO trial, funded by St. Jude Medical, now Abbott), Cleveland Clinic (including for the ExCEED trial, funded by Edwards), Contego Medical (Chair, PERFORMANCE 2), Duke Clinical Research Institute, Mayo Clinic, Mount Sinai School of Medicine (for the ENVISAGE trial, funded by Daiichi Sankyo), Population Health Research Institute; Honoraria: American College of Cardiology (Senior Associate Editor, Clinical Trials and News, ACC.org; Vice chair, ACC Accreditation Committee), Baim Institute for Clinical Research (formerly Harvard Clinical Research Institute; RE-DUAL PCI clinical trial steering committee funded by Boehringer Ingelheim; 
AEGIS-II executive committee funded by CSL Behring), Belvoir Publications (Editor in Chief, Harvard Heart Letter), Duke Clinical Research Institute (clinical trial steering committees, including for the PRONOUNCE trial, funded by Ferring Pharmaceuticals), HMP Global (Editor in Chief, Journal of Invasive Cardiology), Journal of the American College of Cardiology (Guest Editor; Associate Editor), K2P (Co-Chair, interdisciplinary curriculum), Level Ex, Medtelligence/ ReachMD (CME steering committees), MJH Life Sciences, Population Health Research Institute (for the COMPASS operations committee, publications committee, steering committee, and USA national co-leader, funded by Bayer), Slack Publications (Chief Medical Editor, Cardiology Today's Intervention), Society of Cardiovascular Patient Care (Secretary/Treasurer), WebMD (CME steering committees); Other: Clinical Cardiology (Deputy Editor), NCDR-ACTION Registry Steering Committee (Chair), VA CART Research and Publications Committee (Chair); Research funding: Abbott, Afimmune, Amarin, Amgen, AstraZeneca, Bayer, Boehringer Ingelheim, Bristol-Myers Squibb, Cardax, Chiesi, CSL Behring, Eisai, Ethicon, Ferring Pharmaceuticals, Forest Laboratories, Fractyl, Idorsia, Ironwood, Ischemix, Lexicon, Lilly, Medtronic, Pfizer, PhaseBio, PLx Pharma, Regeneron, Roche, Sanofi Aventis, Synaptic, The Medicines Company; Royalties: Elsevier (Editor, Cardiovascular Intervention: A Companion to Braunwald's Heart Disease); Site coinvestigator: Biotronik, Boston Scientific, CSI, St. Jude Medical (now Abbott), Svelte; Trustee: American College of Cardiology; Unfunded research: FlowCo, Merck, Novo Nordisk, Takeda.

Bays' research site has received research grants from 89Bio, Acasti, Akcea, Allergan, Alon Medtech/Epitomee, Amarin, Amgen, AstraZeneca, Axsome, Boehringer Ingelheim, Civi, Eli Lilly, Esperion, Evidera, Gan and Lee, Home Access, Janssen, Johnson and Johnson, Lexicon, Matinas, Merck, Metavant, Novartis, Novo Nordisk, Pfizer, Regeneron, Sanofi, Selecta, TIMI, and Urovant. Bays has served as a consultant/advisor for 89Bio, Amarin, Esperion, Matinas, and Gelesis, and speaker for Esperion.

McCormick, Caldwell, Guerin, Ahmad, Singh, Moreo, Carter, Heggen, and Sapir have nothing to disclose.

\section{ACKNOWLEDGMENTS}

Medical writing support under the authors' guidance was provided by Jeffery Brubaker, $\mathrm{PhD}$, and was funded by PRIME Education.

\section{REFERENCES}

1. Brunner FJ, Waldeyer C, Ojeda F, et al. Application of non-HDL cholesterol for population-based cardiovascular risk stratification: results from the multinational cardiovascular risk consortium. Lancet. 2019;394(10215):2173-83. doi: 10.1016/S0140-6736(19)32519-X

2. Robinson JG, Jayanna MB, Brown AS, et al. Enhancing the value of PCSK9 monoclonal antibodies by identifying patients most likely to benefit. A consensus statement from the National Lipid Association. J Clin Lipidol. 2019;13(4):525-37.

3. Fox KM, Tai M-H, Kostev K, et al. Treatment patterns and low-density lipoprotein cholesterol (LDL-C) goal attainment among patients receiving high- or moderate-intensity statins. Clin Res Cardiol. 2018;107(5):380-88.

4. Cuchel M, Bruckert E, Ginsberg HN, et al. Homozygous familial hypercholesterolemia: new insights and guidance for clinicians to improve detection and clinical management. A position paper from the Consensus Panel on Familial Hypercholesterolemia of the European Atherosclerosis Society. Eur Heart J. 2014;35(32):2146-57.

5. Nordestgaard BG, Chapman MJ, Humphries SE, et al. Familial hypercholesterolaemia is underdiagnosed and undertreated in the general population: guidance for clinicians to prevent coronary heart disease. Eur Heart J. 2013;34(45):3478-90.

6. Raal F, Panz V, Immelman A, Pilcher G. Elevated PCSK9 levels in untreated patients with heterozygous or homozygous familial hypercholesterolemia and the response to high-dose statin therapy. J Am Heart Assoc. 2013;2(2):e000028.

7. Robinson JG, Farnier M, Krempf M, et al. Efficacy and safety of alirocumab in reducing lipids and cardiovascular events. N Eng J Med. 2015;372(16):1489-99.
8. Sabatine MS, Guigliano RP, Keech AC, et al. Evolocumab and clinical outcomes in patients with cardiovascular disease. N Eng J Med. 2017;376(18):1713-22.

9. Hartgers ML, Defesche JC, Langslet G, et al. Alirocumab efficacy in patients with double heterozygous, compound heterozygous, or homozygous familial hypercholesterolemia. J Clin Lipidol. 2018;12(2):390-96.

10. Schwartz GG, Steg PG, Szarek M, et al., for the ODYSSEY OUTCOMES Committees and Investigators.

Alirocumab and cardiovascular outcomes after acute coronary syndrome. N Engl J Med. 2018;379:2097-107.

11. Taskinen MR, Del Prato S, BujasBobanovic M, et al. Efficacy and safety of alirocumab in individuals with type 2 diabetes mellitus with or without mixed dyslipidaemia: Analysis of the ODYSSEY LONG TERM trial. Atherosclerosis. 2018;276:124-30.

12. AlTurki A, Marafi M, Dawas A, et al. Meta-analysis of randomized controlled trials assessing the impact of proprotein convertase subtilisin/kexin type 9 antibodies on mortality and cardiovascular outcomes. Am J Cardiol. 2019;124(12):1869-75.

13. Murphy SA, Pedersen TR, Gaciong ZA, et al. Effect of the PCSK9 inhibitor evolocumab on total cardiovascular events in patients with cardiovascular disease: a prespecified analysis from the FOURIER Trial. JAMA Cardiol. 2019;4(7):613-19.

14. Sarsam S, Berry A, Degheim G, et al. Real-world use of PCSK9 inhibitors: single center experience. J Int Med Res. 2019;47(1):265-70.

15. Praluent (alirocumab) injection, for subcutaneous use. Regeneron/SanofiAventis. September 2020. Accessed November 4, 2020. https://www.regeneron.com/sites/default/files/Praluent_ PI.pdf

16. Repatha (evolocumab) injection, for subcutaneous use. Amgen. May 2020. Accessed November 4, 2020. https:// www.pi.amgen.com/ /media/amgen/ repositorysites/pi-amgen-com/repatha/ repatha_pi_hcp_english1-5.ashx 
17. Grundy SC, Stone NJ, Bailey AL, et al. 2018 AHA/ACC/AACVPR/AAPA/ABC/ ACPM/ADA/AGS/APhA/ASPC/NLA/ PCNA Guideline on the Management of Blood Cholesterol: executive summary. J Amer Coll Cardiol. 2019;73(24):3168-209.

18. Lloyd-Jones DM, Morris PB, Ballantyne CM, et al. 2017 focused update of the 2016 acc expert consensus decision pathway on the role of non-statin therapies for ldlcholesterol lowering in the management of atherosclerotic cardiovascular disease risk. J Am Coll Cardiol. 2017;70(14):1785-822.

19. Hess GP, Natarajan P, Faridi KF, et al. Proprotein convertase subtilisin/kexin type 9 inhibitor therapy. payer approvals and rejections, and patient characteristics for successful prescribing. Circulation. 2017;136(23):2210-19.

20. Cohen JD, Cziraky MJ, Jacobson TA, et al. Barriers to PCSK9 inhibitor prescriptions for patients with high cardiovascular risk: results of a healthcare provider survey conducted by the National Lipid Association. J Clin Lipidol. 2017;11(4):891-900.

21. Chamberlain AM, Gong Y, Shaw KM, et al. PCSK9 inhibitor use in the real world: data from the National PatientCentered Research Network. J Am Heart Assoc. 2019;8(9):e011246.

22. Alakbarzade V, Pereira AC. What proportion of patients admitted with stroke or transient ischemic attack may be suitable for newer cholesterol-lowering treatment? J Stroke Cerebrovasc Dis. 2020;29(1):104457.

23. Myers KD, Farboodi N, Mwamburi M, et al. Effect of access to prescribed PCSK9 inhibitors on cardiovascular outcomes. Circ Cardiovasc Qual Outcomes. 2019;12(8):e005404.

24. Doshi JA, Puckett JT, Parmacek MS, Rader DJ. Prior authorization requirements for proprotein convertase subtilisin/kexin type 9 inhibitors across US private and public payers. Circ Cardiovasc Qual Outcomes. 2018;11(1):e003939.
25. Baum SJ, Toth PP, Underberg JA, et al. PCSK9 inhibitor access barriers-issues and recommendations: improving the access process for patients, clinicians and payers. Clin Cardiol. 2017;40:243-54.

26. AMCP Partnership Forum: optimizing prior authorization for appropriate medication selection. J Manag Care Spec Pharm. 2020;26(1):55-62. doi: 10.18553/ jmcp.2020.26.1.55

27. Ritchey MD, Wall HK, Owens PL, Wright JS. Vital signs: state-level variation in nonfatal and fatal cardiovascular events targeted for prevention by Million Hearts 2022. MMWR Morb Mortal Wkly Rep. 2018;67(35):974-82. doi: http://dx.doi. org/10.15585/mmwr.mm6735a3

28. U.S. Census Bureau. City and town population totals: 2010-2019. Accessed October 26, 2020. https://www.census. gov/data/tables/time-series/demo/ popest/2010s-total-cities-and-towns. $\underline{\mathrm{html}}$

29. Bhatt DL, Briggs AH, Reed SD, et al. Cost-effectiveness of alirocumab in patients with acute coronary syndromes: The ODYSSEY OUTCOMES trial. J Am Coll Cardiol. 2020;75(18):2297-308.

30. Steg PG, Szarek M, Bhatt DL, et al. Effect of alirocumab on mortality after acute coronary syndromes: an analysis of the ODYSSEY OUTCOMES randomized clinical trial. Circulation. 2019;140(2):103-12.

31. Bays HE. Alirocumab, decreased mortality, nominal significance, $P$ values, Bayesian statistics, and the duplicity of multiplicity. Circulation. 2019;140(2):113-16.

32. Handelsman Y, Jellinger P, Guerin C, et al. Consensus statement by the American Association of Clinical Endocrinologists and American College of Endocrinology on the management of dyslipidemia and prevention of cardiovascular disease algorithm - 2020 executive summary. Endocr Pract. 2020;26(10):1196224. doi: $10.4158 / C S-2020-0490$
33. Mach F, Baigent C, Catapano AL, et al. 2019 ESC/EAS Guidelines for the management of dyslipidaemias: lipid modification to reduce cardiovascular risk: the Task Force for the Management of Dyslipidaemias of the European Society of Cardiology (ESC) and European Atherosclerosis Society (EAS). Eur Heart J. 2020;41(1):111-88.

34. Reynolds VW, Chinn ME, Jolly JA, et al. Integrated specialty pharmacy yields high PCSK9 inhibitor access and initiation rates. J Clin Lipidol. 2019;13(2):254-64.

35. Gencer B, Koskinas KC, Raber L, et al. Eligibility for PCSK9 inhibitors according to American College of Cardiology (ACC) and European Society of Cardiology/European Atherosclerosis Society (ESC/EAS) Guidelines After Acute Coronary Syndromes. J Am Heart Assoc. 2017;6(11):e006537.

36. Lloyd-Jones DM, Morris PB, Ballantyne CM, et al. 2016 ACC expert consensus decision pathway on the role of non-statin therapies for LDL-cholesterol lowering in the management of atherosclerotic cardiovascular disease risk. J Am Coll Cardiol. 2016;68(1):92-125.

37. Maciejko JJ, Jamoua R, Anne P. Assessment and management of patients with hyperlipidemia referred for initiation of PCSK9 inhibitor therapy: a lipid clinic experience. Am J Cardiovasc Drugs. 2019;19(6):553-59.

38. Zamora A, Masana L, Comas-Cufi M, et al. Number of patients eligible for PCSK9 inhibitors based on real-world data from 2.5 million patients. Rev Esp Cardiol. 2018;71(12):1010-17. 